\title{
Thermosolutal Convection in a Porous Medium Cavity Subjected to Heat and Mass Flux: A Discrete Fracture Effect
}

\author{
Zakaria AOUF $^{1}$, Chakib SELADJI ${ }^{1}$ \\ ${ }^{1}$ Energetic and Applied Thermal Laboratory (ETAP), Faculty of Technology, \\ Aboubekr belkaid University, Tlemcen, 13000, Algeria; \\ zakaria.aouf@mail.univ-tlemcen.dz / c_seladji@mail.univ-tlemcen.dz
}

Manuscript received November 26, 2019; revised October 11, 2020

\begin{abstract}
Species separation in heterogeneous porous media is a field of interest of many industrial activities. In our investigation, the effect of a single discrete fracture on the thermosolutal convection coupled with the Soret effect have been analyzed. The main results show that the fracture can greatly affect the behavior of the thermogravitational flow and might play a positive role to the separation caused by the Soret effect. Furthermore, the fracture tilted to the cold wall causes a large separation compared to the one tilted to the hot wall with the same angle. Therefore, the separation process could be greatly improved.
\end{abstract}

Keywords: Soret effect; thermodiffusion; permeability; fracture; separation.

\section{Nomenclature}

$\boldsymbol{A} \quad$ aspect ratio of the porous matrix, $\boldsymbol{H}^{\prime} / \boldsymbol{L}^{\prime}$

a Rayleigh ratio, $\boldsymbol{R}_{T F} / \boldsymbol{R}_{T P M}$

$\boldsymbol{C}$ dimensionless solute concentration, $\left(\boldsymbol{C}^{\prime}-\boldsymbol{C}_{\mathbf{0}}^{\prime}\right) / \Delta \boldsymbol{C}^{\prime}$

$\boldsymbol{C}_{\mathbf{0}}^{\prime} \quad$ reference solute concentration

$\Delta \boldsymbol{C}^{\prime} \quad$ characteristic solute concentration, $\boldsymbol{j}^{\prime} \boldsymbol{H}^{\prime} / \boldsymbol{D}$

D mass diffusivity of species

$\boldsymbol{D}_{\boldsymbol{T}} \quad$ thermodiffusion coefficient

$\boldsymbol{e} \quad$ aperture of the fracture, $\boldsymbol{e}^{\prime} / \boldsymbol{H}^{\prime}$

$\overrightarrow{\mathbf{g}}$ gravitational acceleration vector, $\mathrm{m} / \mathrm{s}^{2}$

$\boldsymbol{H}^{\prime} \quad$ height of the cavity, $\mathrm{m}$

$\overrightarrow{\mathbf{J}^{\prime}} \quad$ constant mass flux, mole $/ \mathrm{m}^{2} / \mathrm{s}$

$\boldsymbol{K}$ permeability of the porous medium

$\boldsymbol{K}_{\boldsymbol{F}} \quad$ permeability of the fracture

$\boldsymbol{L}^{\prime} \quad$ width of the cavity, $\mathrm{m}$ 


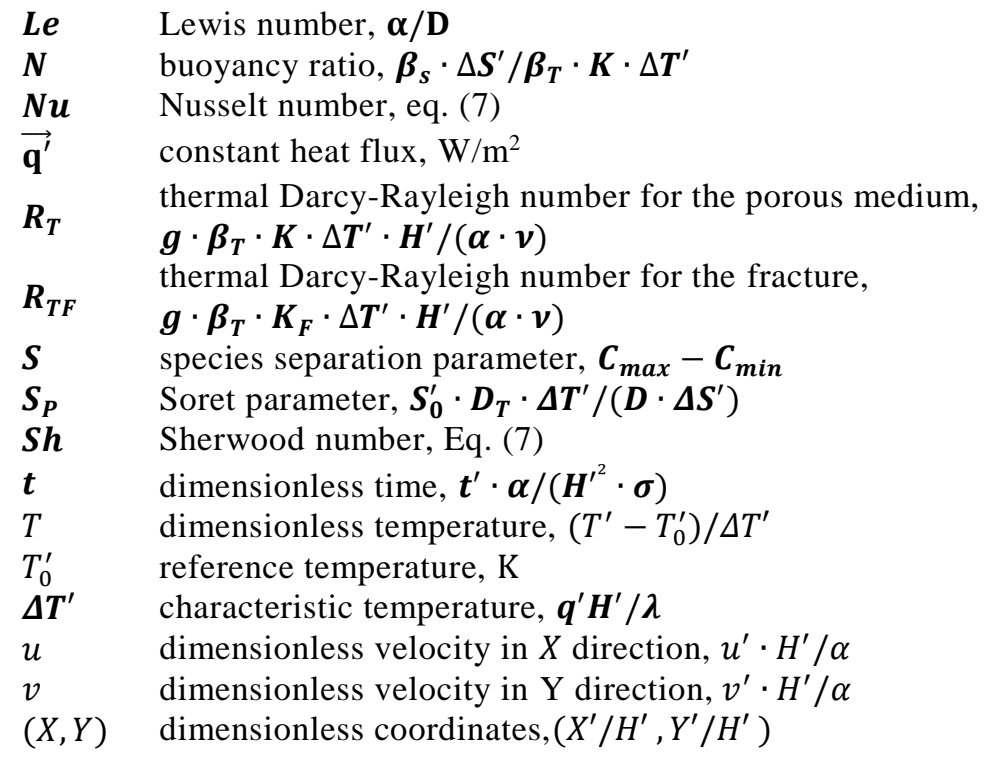

\section{Greek Symbols}

$\begin{array}{ll}\alpha & \text { thermal diffusivity } \\ \beta_{S} & \text { solute concentration expansion coefficient } \\ \beta_{T} & \text { thermal expansion coefficient } \\ \varepsilon & \text { normalized Porosity, } \varepsilon^{\prime} / \sigma \\ \varepsilon^{\prime} & \text { porosity of the porous medium } \\ \lambda & \text { thermal conductivity, W/m/K } \\ v & \text { kinematic viscosity of the fluid } \\ \rho & \text { density of the fluid mixture } \\ (\rho c)_{F} & \text { heat capacity of the fluid mixture } \\ (\rho c)_{P M} & \text { heat capacity of the saturated porous medium } \\ \sigma & \text { heat capacity ratio, }(\rho c)_{P M} /(\rho c)_{F} \\ \psi & \text { dimensionless stream function, } \psi^{\prime} / \alpha\end{array}$

\section{Superscripts}

' for dimensional variable

Subscripts
$F \quad$ Fracture
$P M \quad$ porous medium
Opt refers to optimum value
Max refers to maximum value
Min refers to minimum value
0 refers to Reference state 
$\begin{array}{ll}S & \text { refers to solutal } \\ T & \text { refers to thermal }\end{array}$

\section{Introduction}

Due to the thermodiffusion effect [1], a mass fraction gradient is spontaneously created when a liquid or gas mixture is subjected to a temperature difference. The thermodiffusion effect, also called Soret effect [2], or LudwigSoret effect [3] causes a separation of components, where generally the heavier or larger molecular species concentrate near the colder surface. Coupling of this Soret effect with natural convection magnifies the primary separation coming from the Soret effect. This is known as the thermogravitational phenomenon. Clusius and Dickel [4] invented the thermogravitational column (TGC) in order to separate isotopes, which was developed theoretically by Furry et al. [5] and extended for liquids by DeGroot [6]. However, the developed TGC was declined due to the operating features and so researchers are encouraged to combine the TGC technique with other separation mechanisms and modified cell designs [7]. Lorenz and Emery [8] promoted the idea of filling the thermogravitational cells with a porous medium, which allows working with large dimension cells. In this regard, many works have recently been carried out to buttress these phenomena. For instance, Lee et al. [9] investigated the effect of various forms of non-uniform basic temperature gradients on thermogravitational convection in an isotropic porous layer using non-equilibrium models. Two-field temperature models were used for the energy equation and the Forchheimer-extended Darcy model to describe the flow. It is found that the linear temperature profile provides a reinforcement of stability, compared with the non-uniform temperature gradients. Abahri et al. [10] studied the thermogravitational separation of a binary liquid mixture in a horizontal porous annulus space heated isothermally from the inner cylinder. Theoretical and numerical results reveal that the separation can be increased for small values of Rayleigh number. Grigor'ev and Rivin [11] used the thermogravitational column to determine thermodiffusion factors of organic liquids mixture. A comparison of the calculated values of the Soret coefficient and the experimental data obtained has been presented with a satisfactory agreement. In terms of the separation ratio, and the cross-diffusion, Lewis and Rayleigh numbers, Mutschler et al. [12] suggests two analytical models to describe the thermogravitational separation of $\mathrm{N}$-component mixtures in a vertical cavity filled by a porous medium. Their theoretical model supports strongly the experimental results. Recently, Mojtabi et al. [13] studied the influence of the thickness and the nature of the bounding horizontal plates on the separation of a binary mixture in a horizontal porous cavity. Authors found that the ratio of the plate to the porous layer thickness and the ratio of their thermal 
conductivity greatly affect the species separation. The analytical results were confirmed by direct numerical simulations. Kozlova and Ryzhkov [14], studied the transient separation of a multicomponent mixture in a cylindrical thermogravitational column. The evaluation of separation in vertical direction is solved analytically and compared with two-dimensional numerical simulation of binary and ternary mixture, where a good agreement is observed. Hashemipour et al. [15] performed an experimental and numerical study to separate toluene/nheptane mixture by TGC. Good concordance can be seen between experimental and numerical results. The separation factor can reach 1.17 when the optimal conditions are achieved (feed flow rate, cut, temperature gradient). In a packed thermogravitational cell, maximum separation is associated with an optimal permeability of porous medium as it was a subject of interest to most researchers [16], [17].

Thermosolutal convection in vertical porous cavity can be driven either by double diffusion (the solute gradient is induced by the solute boundary condition), or by cross diffusion (the solute gradient is induced by thermal boundary condition via Soret effect) [18]. However, in many practical applications, the thermosolutal convection is driven by both double diffusive convection and Soret effect [19]. In this wise, Amahmid et al. [20] studied the double diffusive convection in a vertical cavity subjected to horizontal gradient of heat and mass. They focused on the situation in which thermal and solutal buoyancy forces are opposed to each other and found that multiple steady state solutions are possible. Er-Raki et al. [21] studied the Soret effect on double diffusive boundary layer flowing in a vertical porous layer. Based on the parallel flow approximation, an analytical solution was developed which allows a detailed parametric study of the governing parameters effect on the convective flow and on heat and mass transfer characteristics. These analytical results were compared with the numerical solution. Jiang et al. [22] examined numerically the effect of natural convection on the Soret effect in a vertical cavity heated laterally. They used the mathematical model of Shukla and Firoozabadi to calculate molecular diffusion, thermodiffusion, and pressure diffusion coefficients as functions of temperature, pressure, and other physical properties of the binary mixture at each point of the grid. As a function of separation factor, their results indicate that as permeability increases, separation increases until it peaks at $10 \mathrm{md}$ (md: millidarcy, a non-S.I. unit of the permeability), then decreases. Jiang et al. [23] examined using the same mathematical model the effect of medium heterogeneity in a stratified porous vertical cavity. They found that the Soret effect is strongly dependent on medium heterogeneity; the authors announced that the separation in heterogeneous porous medium is always greater than in homogeneous one. Their declaration is further elaborated in the results section of this paper. Jaber [24] thereafter studied the effect of the permeability in a fractured porous medium 
cavity filled with a ternary mixture with the consideration of likely thermodiffusion effect. The system was set at $10 \mathrm{md}$ for the permeability of the porous medium, while the permeability of the fracture was varied from 10 to 1000 md. The outcome showed an increasing of the flow field in the fracture, which affects the behavior of each component.

Limited studies considered the Soret effect while investigating the thermosolutal convection phenomenon in heterogeneous porous media. This study has the objective of investigating the role of a discrete fracture on thermosolutal convection coupled with the Soret effect in an isotropic porous media saturated by a binary fluid. The fracture has been considered as another porous medium with slightly higher permeability. More precisely, the influence of the fracture characteristics (permeability, positioning, thickness) on the fluid flow and heat and mass transfer is examined in terms of the separation parameter " $S$ ", which is defined as the difference of the maximum concentration in the hot wall and the minimum concentration in the cold wall [25].

\section{Problem formulation}

The problem studied in this paper is performed using a numerical finite difference method. Within a rectangular cavity, the thermosolutal convection is coupled with the Soret effect in a two-dimensional fractured porous media saturated by a binary fluid. The same configuration is used as the one presented in [21]. Neumann boundary conditions for solute and temperature are applied to the vertical walls of the cavity such that it is heated and salted from the right-end, while the horizontal walls are adiabatic and impermeable (Fig. 1). Darcy's law is governing the flow which is assumed laminar and incompressible. The thermophysical properties of the mixture are considered constant. Note that for this study, the Soret parameter is assumed constant, the negligible effect of the mass concentration variation on the heat transfer (Dufour effect) is not considered. Using the Boussinesq approximation, the dimensionless governing equations of the mathematical model are as follows [26]:

$$
\begin{gathered}
\frac{\partial^{2} \psi}{\partial x^{2}}+\frac{\partial^{2} \psi}{\partial y^{2}}=-R_{T} \cdot\left(\frac{\partial T}{\partial x}+N \cdot \frac{\partial C}{\partial x}\right), \\
\varepsilon \cdot \frac{\partial C}{\partial t}+\frac{\partial(u \cdot C)}{\partial x}+\frac{\partial(v \cdot C)}{\partial y}=\frac{1}{L e} \cdot\left(\nabla^{2} C+S_{P} \cdot \nabla^{2} T\right) \\
\frac{\partial T}{\partial t}+\frac{\partial(u T)}{\partial x}+\frac{\partial(v T)}{\partial y}=\nabla^{2} T .
\end{gathered}
$$




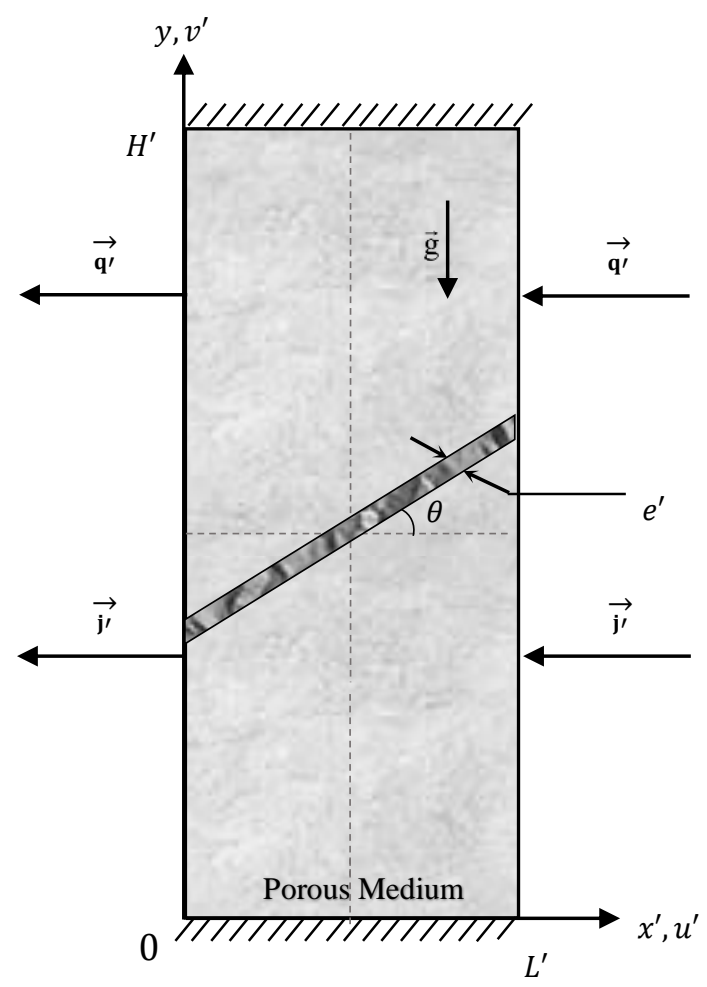

Figure 1: Geometry of the physical system

Introducing the stream function (the flow velocity components can be expressed as the derivatives of the scalar stream function), the dimensionless velocity components are calculated as follows:

$$
u=\frac{\partial \psi}{\partial y} ; \quad v=-\frac{\partial \psi}{\partial x} .
$$

The non-dimensional governing parameters appearing in the equations are the thermal Darcy-Rayleigh number, $R_{T}$, the Lewis number $L e$, the buoyancy ratio $N$, the Soret parameter, $S p$, and the aspect ratio, $A$. They are defined in equation 5 .

$$
\begin{gathered}
R_{T}=\frac{g \beta_{T} K \Delta T^{\prime} H^{\prime}}{\alpha \nu} ; \quad L e=\frac{\alpha}{D} ; \quad N=\frac{\beta_{S} \Delta C^{\prime}}{\beta_{T} \Delta T^{\prime}} . \\
\varepsilon=\frac{\varepsilon^{\prime}}{\sigma} ; \quad S p=\frac{D_{T} C_{0}^{\prime} \Delta T^{\prime}}{D \Delta C^{\prime}} ; \quad A=\frac{H^{\prime}}{L^{\prime}}=5
\end{gathered}
$$


Note, that the classical separation ratio $\varphi$ is related to $S_{p}$ and $N$ by the relation $S p=\varphi / N$ [26]. In terms of dimensionless variables, the boundary conditions are defined in (6) [21].

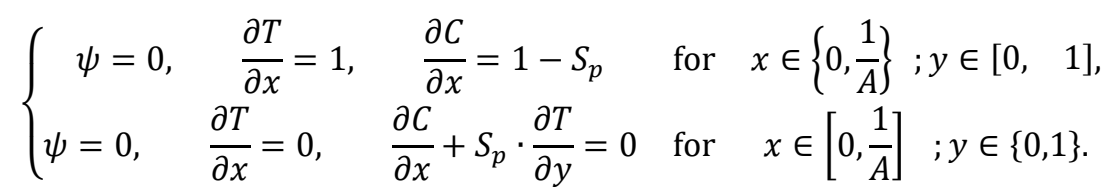

The heat and mass transfer rates, quantified in terms of the Nusselt and Sherwood numbers, can be calculated from the following expressions:

$$
\begin{aligned}
& N u=\frac{1}{\nabla T}=\frac{1}{T\left(L^{\prime}, H^{\prime} / 2\right)-T\left(0, H^{\prime} / 2\right)} \\
& S h=\frac{1}{\nabla C}=\frac{1}{C\left(L^{\prime}, H^{\prime} / 2\right)-C\left(0, H^{\prime} / 2\right)}
\end{aligned}
$$

The mathematical model is applied to the entire cavity. Moreover, a specific permeability is applied to the fracture, which is expressed by a different Rayleigh number $\left(R_{T F}\right)$.

\section{Numerical method}

The governing equations are discretized using a central finite difference scheme and solved with the alternate direction implicit (ADI) method, using a (Fortran 90) numerical code software. A first order forward scheme is used for the transitory term in the Darcy equation, which is solved with the successive over relaxation (SOR) method. A second order forward or backward scheme is used for Neumann boundary conditions. The results are obtained when the system satisfies the convergence criteria (the relative error corresponding to $T, C$, and $\Psi$ between successive time steps for each node is less than $10^{-9}$ ). The effect of the grid size on the results was analyzed using uniform rectangular mesh. Table 1 indicates satisfying results when using a grid with at least $171 \times 171$ nodes. The maximum relative errors for $\mathrm{Nu}, \mathrm{Sh}$, and $S$ do not exceed $1.5 \%$. 
Table 1: Effect of the grid size for $R_{T}=50, R_{T F}=150, L e=10, S_{p}=0.5$, and $\theta=70$.

\begin{tabular}{c|cccc}
\hline Grid & Nu & $S h$ & $S$ & Relative error \% \\
\hline $71 \times 71$ & 2.5400 & 11.59 & 0.3837 & 4.93 \\
$101 \times 101$ & 2.5430 & 11.8 & 0.3916 & 2.97 \\
$131 \times 131$ & 2.5480 & 11.91 & 0.3956 & 1.98 \\
$171 \times 171$ & 2.5510 & 11.97 & 0.3976 & 1.48 \\
$301 \times 301$ & 2.5540 & 12.05 & 0.4015 & 0.52 \\
$501 \times 501$ & 2.5548 & 12.09 & 0.4035 & 0.02 \\
$601 \times 601$ & 2.5549 & 12.09 & 0.4036 & 0 \\
\hline
\end{tabular}

\section{Results and analysis}

The aim of this study is to understand the effects of a discrete fracture on heat and mass transfer in porous media. More attention is given to determine the optimum fracture conditions that gives a higher separation for an aspect form $A=5$, and a buoyancy ratio $N=0.4$. To achieve this objective, an inclined fracture passed across the center of the cavity was established as depicted in Fig. 1. The latter is characterized by an angle $\theta$, an aperture $e$, and a specific Rayleigh number $R_{T F}$ as determined by the fracture permeability. Furthermore, the ratio of the fracture Rayleigh number $R_{T F}$ over the porous medium Rayleigh number $R_{T}$ is calculated and denoted as, $\mathrm{a}=R_{T F} / R_{T}$. For all simulations, a mono-cellular flow occurred in the cavity.

\subsection{Validation}

The mathematical model and resolution process need to be validated using a known case. For this purpose, the boundary conditions were modified to reproduce the results of Khadiri et al. [26] in which the authors studied the Soret effect on double-diffusive convection in a square porous cavity heated and salted from the bottom Fig. 2 shows the comparison of the concentration profiles in the mid-width of the cavity corresponding to a bi-cellular flow obtained with different values of Soret parameter $S_{p}$ from this study and that of [26], good agreement could be seen. Results show that at $Y=0.18$, the concentration remains unchanged when $S_{p}$ is varied. This behavior was denominated by [26] as a neutral position and has not been explained. However, using a small Rayleigh number $R_{T}=50$ (for example), the Soret effect becomes more important and dominates convection forces, for which these neutral positions disappear. This outcome is shown in Fig. 3 even though such results were not presented by [26]. 


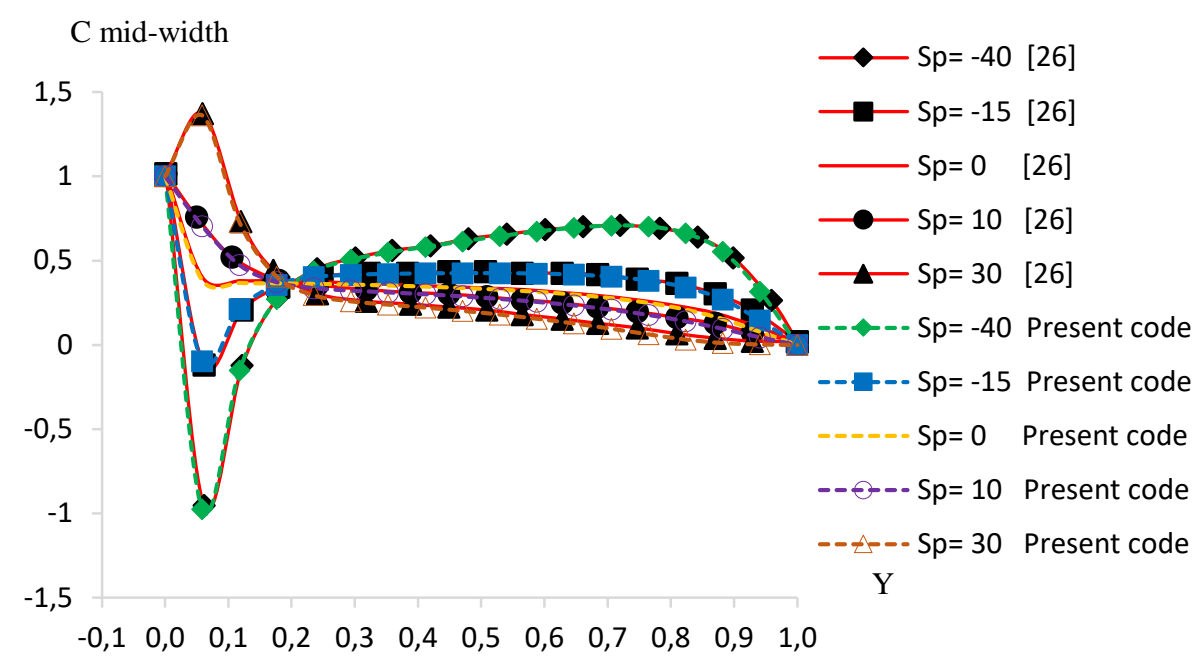

Figure 2: Vertical profiles of concentration at $X=0.5$ for $A=1, R_{T}=200, L e=10, N=0.1$, and various values of $S p$

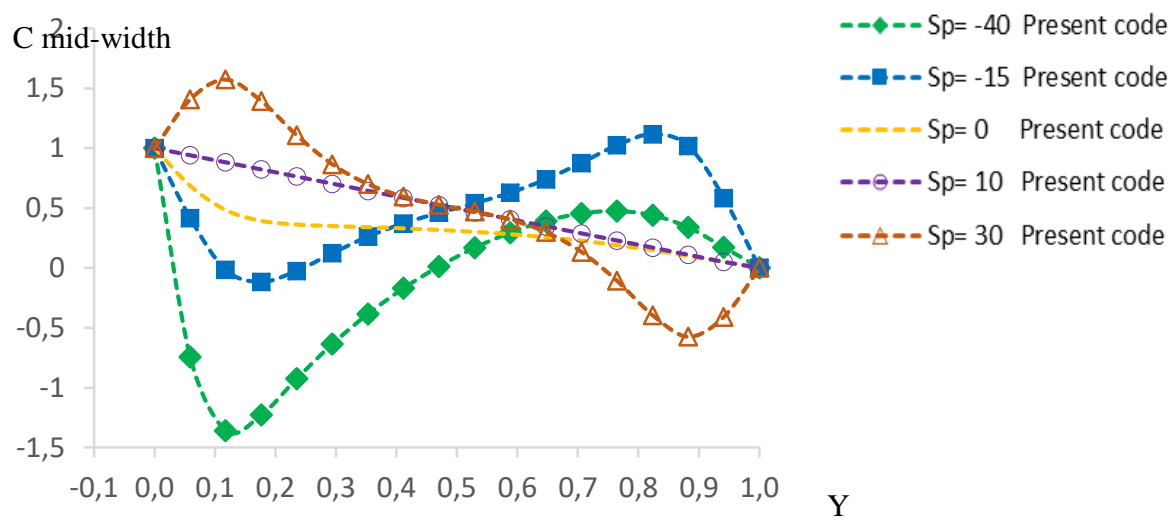

Figure 3: Vertical profiles of concentration at $X=0.5$ for $A=1, R_{T}=50, L e=10$, $N=0.1$, and various values of $S_{p}$ 


\subsection{Soret parameter effects}

The contribution of the convection with the Soret coefficient depending on the permeability of the medium can lead to a higher separation of the mixture constituents. The effect of Soret parameter is investigated for an extent Rayleigh number scope. Fig. 4 shows that the separation for all the Soret parameter has the same pattern, in which three different zones are distinguished: at low Rayleigh number $\left(R_{T}<0.01\right)$ the permeability of the porous medium is low, and only diffusion and thermodiffusion effects cause the mass transfer (purely diffusive regime). Augmenting $R_{T}$ leads to growth in convection forces, the contribution of the convection forces with the Soret effect performs the peak of the separation at an optimal Rayleigh number $R_{\text {Topt }}$ and creates a diagonal concentration gradient in the cavity. At high values of Rayleigh number, convection forces dominate the process, mixing the constituents of the binary fluid, where thermodiffusion is considered as a secondary phenomenon displays no significant effect, and the separation in this zone is less than in purely diffusive regime zone. Furthermore, the separation is also affected by the Soret parameter sign. Generally, for positive Soret value, the bigger and heavier components migrate to the cold wall of the cavity (left end) and might give a negative Sherwood number. However, for negative value of Soret parameter, thermodiffusion effect is opposing diffusion effect (in our case), were the heavier component moves towards the hot wall of the cavity (right-end) and withhold the gradient of concentration, which explains the higher separation values. More results are shown in Table 2. For $\mathrm{R}_{\mathrm{T}}=0.01$, the gradient of concentration is made horizontally and (the difference of concentration calculated at the middle of the cavity) is almost the same as S, but for $\mathrm{R}_{\mathrm{T}}=1$, the concentration gradient is diagonal, and the separation becomes more important. Note that depending on the reference value in the dimensionless process, the separation can be higher than 1 .

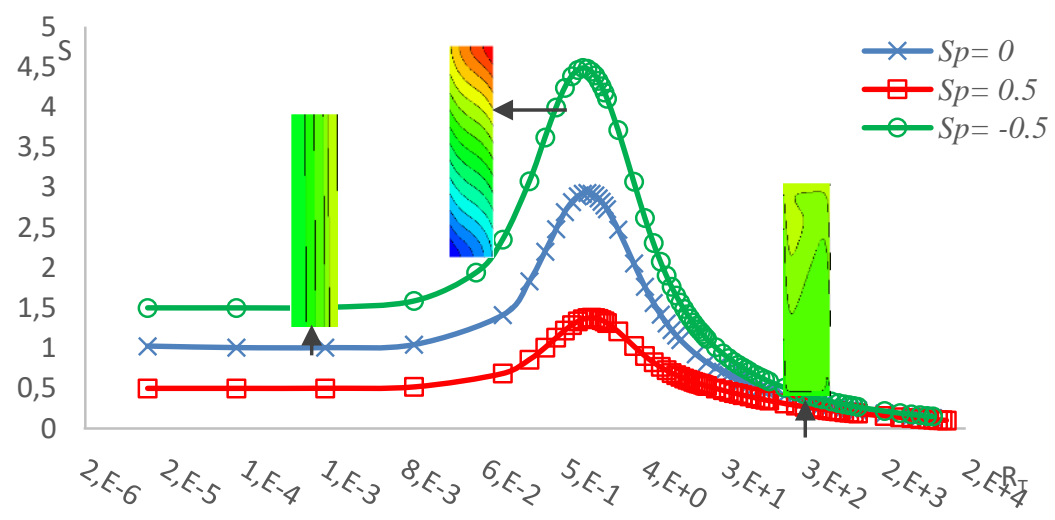

Figure 4: Separation as function of $R_{T}$ for $L e=10, N=0.4$, and various values of $S p$ 
Table 2. Effect of Soret parameter ( $S p)$ sign at different $R_{T}$ for $L e=10$ and homogeneous porous medium

\begin{tabular}{c|rcccrrr}
\hline \multirow{3}{*}{$R_{T}$} & $S p$ & $\psi_{\text {Min }}$ & $\psi_{\text {Max }}$ & $N u$ & \multicolumn{1}{c}{$S h$} & $\nabla C=\frac{1}{S h}$ & \multicolumn{1}{c}{$S$} \\
\hline \multirow{5}{*}{0.01} & -5 & $0.0 \mathrm{E}+00$ & $4.2 \mathrm{E}-04$ & 1.000 & 0.167 & 5.995 & 6.792 \\
& -2 & $0.0 \mathrm{E}+00$ & $2.7 \mathrm{E}-04$ & 1.000 & 0.335 & 2.985 & 3.253 \\
& 0 & $0.0 \mathrm{E}+00$ & $1.7 \mathrm{E}-04$ & 1.000 & 1.000 & 1.000 & 1.005 \\
& 2 & $0.0 \mathrm{E}+00$ & $7.5 \mathrm{E}-04$ & 1.000 & -1.000 & -1.000 & 1.024 \\
& 5 & $-7.5 \mathrm{E}-04$ & $0.0 \mathrm{E}+00$ & 1.000 & -0.250 & -4.000 & 4.093 \\
\hline \multirow{4}{*}{1} & -5 & $0.0 \mathrm{E}+00$ & $1.9 \mathrm{E}-01$ & 1.018 & 0.446 & 2.242 & 17.139 \\
& -2 & $0.0 \mathrm{E}+00$ & $1.7 \mathrm{E}-01$ & 1.013 & 0.748 & 1.337 & 8.859 \\
& 0 & $0.0 \mathrm{E}+00$ & $1.5 \mathrm{E}-01$ & 1.009 & 1.803 & 0.555 & 2.923 \\
& 2 & $0.0 \mathrm{E}+00$ & $9.4 \mathrm{E}-02$ & 1.004 & -1.470 & -0.680 & 3.121 \\
& 5 & $-4.9 \mathrm{E}-02$ & $0.0 \mathrm{E}+00$ & 1.001 & -0.281 & -3.559 & 9.691 \\
\hline \multirow{4}{*}{450} & -5 & $0.0 \mathrm{E}+00$ & $2.6 \mathrm{E}+00$ & 4.884 & 6.336 & 0.158 & 0.818 \\
& -2 & $0.0 \mathrm{E}+00$ & $3.0 \mathrm{E}+00$ & 5.523 & 7.703 & 0.130 & 0.515 \\
& 0 & $0.0 \mathrm{E}+00$ & $3.4 \mathrm{E}+00$ & 5.809 & 14.870 & 0.067 & 0.296 \\
& 2 & $0.0 \mathrm{E}+00$ & $3.8 \mathrm{E}+00$ & 5.611 & -13.990 & -0.071 & 0.110 \\
& 5 & $-3.2 \mathrm{E}-01$ & $5.9 \mathrm{E}+00$ & 5.008 & -2.191 & -0.456 & 1.542 \\
\hline
\end{tabular}

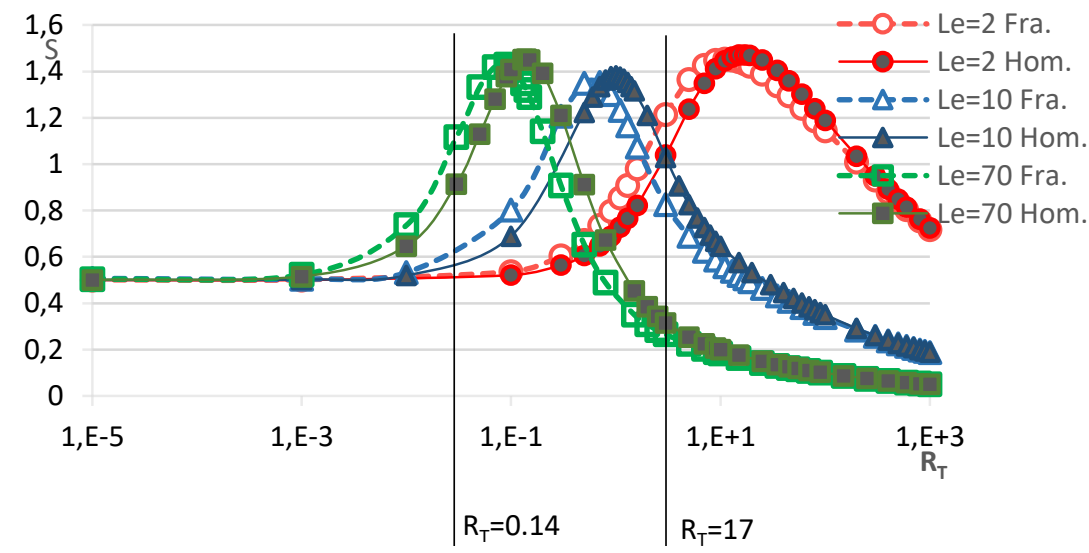

Figure 5. Separation in fractured PM vs. Homogeneous PM for $\theta=70^{\circ}, S_{p}=0.5$, $\alpha=30$, and different values of $L e$ 


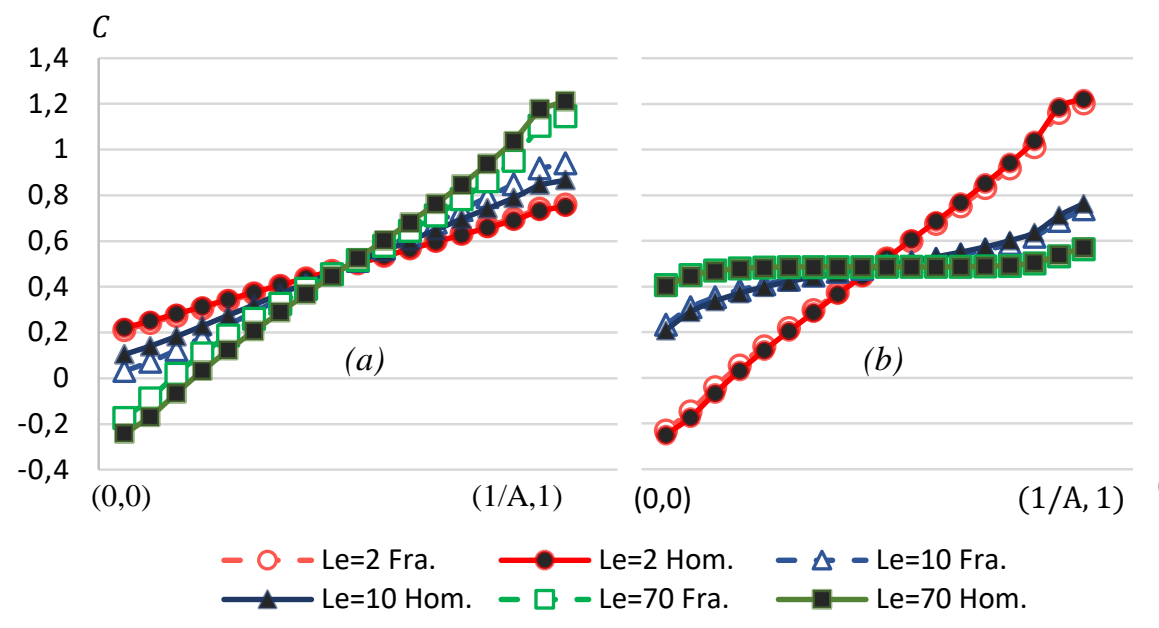

Figure 6. Diagonal profiles of concentration (with \& without Fracture) for $L e=2,10,70$ at $(a) R_{T}=0.14,(b) R_{T}=17$

\subsection{Effect of the Lewis number}

Fig. 5 shows the separation parameter $S$ for different value of $L e$ for an inclined fracture of $\theta=70^{\circ}, e=0.038$, and a Rayleigh ratio $a=30$, solid lines correspond to homogeneous porous medium (HPM), and dotted ones correspond to heterogeneous (fractured) porous medium (FPM). It is clearly demonstrated that the more the Lewis number is increasing $(L e)$, the convection forces becomes more effective, which moves forward the optimal Rayleigh number $R_{T o p t}$, and makes the peak of separation more pointed. Similar results were found by [27] in horizontal cavity.

The fracture behavior towards different $L e$ values is almost the same, adding a fracture (characterized by a higher permeability) to the porous medium, leads to improving the entire permeability of the medium, which favors more the convection forces, and gives a better separation $S$ as long as is less than $R_{T o p t}$. For Rayleigh numbers $R_{T}$ more than $R_{\text {Topt }}$, the fracture decreases the separation. However, a slight difference is observed for higher Lewis numbers, the fracture has more negative effect on the separation when Rayleigh number $R_{T}$ is more than $R_{\text {Topt }}$. This could be referred to the nature of the fluid, in the literature $L e=2$ for gas [27], $L e=10,70$ for liquid [28]. 
Fig. 6 shows the concentration profiles at the diagonal for homogenous and fractured porous media for a fracture of $\theta=70^{\circ}, e=0.038$, and Rayleigh ratio $a=30$. At the center of the cavity, the mixture persists homogeneous for different Rayleigh and Lewis numbers, deviation is more observed in the cavity extremes. The optimal Rayleigh number for $L e=70$ is $R_{T}=0.14$, what explains a higher concentration gradient for $L e=70$ as seen in Fig. $6(a)$, for $L e=10$, the fracture plays a positive role, raising the total permeability of the medium and augmenting the separation $S$ by $19.2 \%$. However, for $L e=2$, and $R_{T}=0.14$, there are no convection forces in the cavity, the fracture has no significance effect, and the concentration profiles represent the boundary conditions effect. Fig. $6(b)$ shows a higher concentration gradient for $L e=2$, since $R_{T}=17$ corresponding to the optimum. However, for $L e=10$, and $70, R_{T}=17$ is higher than the optimal, and the convection forces cause a homogeneous mixture.

\subsection{The fracture's permeability effects}

In order to better understand and confirm the effect of the fracture's permeability on the separation, different Rayleigh numbers were set to the fracture $R_{T F}$, for different values of porous medium Rayleigh number $R_{T}$. Three different values of $\mathrm{R}_{\mathrm{T}}$ were investigated vis-à-vis; less than, equal, and higher than the optimum correspond to $(a),(b)$, and (c) respectively (Fig. 7). Comparing the separation caused by the fracture with that caused by the homogeneous porous medium, when the porous medium permeability is less than the optimal $(a)$, the fracture plays an important role, raising the separation by $93 \%$ at its peak $R_{T}=3$, then it starts decreasing. However, when the porous medium's permeability is higher than or equal to the optimal as in $(b)$ and $(c)$, the fracture can apply a negative effect on the separation. The fracture existence strongly affects the behavior at the optimal Rayleigh number $R_{\text {opt }}$. For a heterogeneous porous media, it was stated by [23] that due to heterogeneity condition of the problem, the separation ratio in heterogeneous porous media is always higher than that in homogeneous porous media. However, throughout these results, their statement is valid when the total porous medium permeability is less than the optimal, and this does not contradict with their results. 

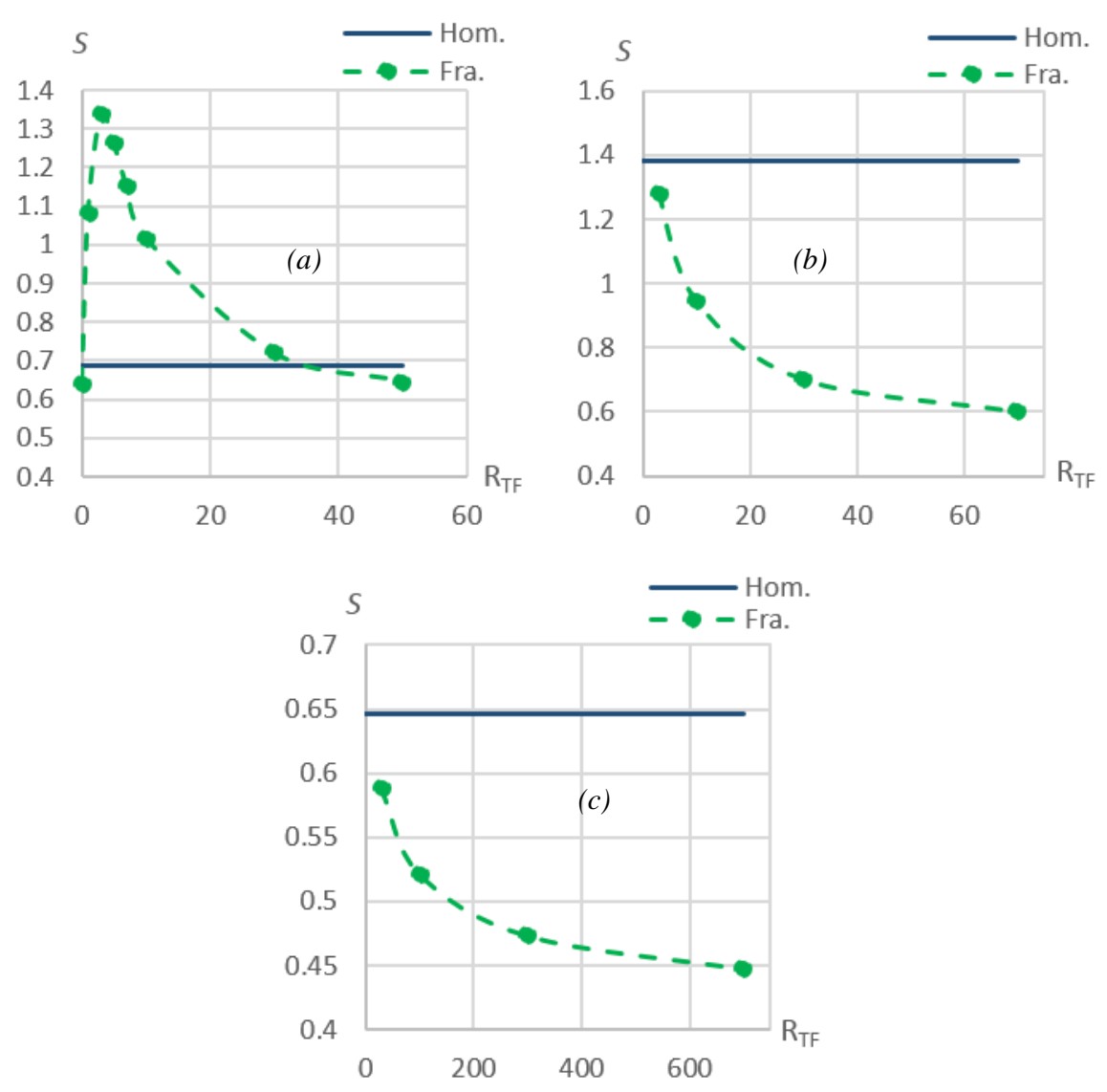

Figure 7: Effect of the fracture's permeability on the separation for $L e=10, \theta=90^{\circ}$, $e=0.038$, and different Rayleigh numbers $(a) R_{T}=0.1$, (b) $R_{T}=1$, (c) $R_{T}=10$

\subsection{The fracture's orientation ( $\theta$ ) effect}

Separation of component by Soret effect becomes more important when it is accompanied by low convection forces. For a better understanding of how these two effects contribute together, a special attention is given to the region that follows the onset of the convection forces. We traced the separation at low Rayleigh number $\left(R_{T}\right)$ for $e=0.038, S_{p}=0.5, L e=10$, and $\alpha=30$ for different fracture's angles (Fig. 8). The results show that for $R_{T}$ less than 0.5 , the separation in fractured porous media is always better than in homogeneous ones. As predicted, fractures precede the optimal $R_{T}$ for the separation whatever the angle. However, comparing vertically symmetrical fractures, curves are identical for $\theta=70^{\circ}$ and $\theta=110^{\circ}$, but they become different for angles near the horizontal. In 
order to understand the main cause of this variance, the heat and mass transfer represented by Nusselt and Sherwood numbers are presented in Fig. $9(a)$ and (b) respectively. For a vertical fracture, the mass transfer is almost the same as in homogeneous porous medium (without fracture) with some slight decrease, while the horizontal fracture significantly increases it. As explained in [29], the horizontal dynamics in the Clusius and Dickel thermogravitational column is governed by Soret effect, and at low Rayleigh number $R_{T}$ weak convection forces exist, thus, the mass transfer is made horizontally by diffusion and thermodiffusion effects. Therefore, the more the fracture is inclined towards the horizontal, the higher mass transfer is. The same observation can be seen for Nusselt number (Fig. $9(a))$ since the same boundary condition are applied for heat and mass transfer.

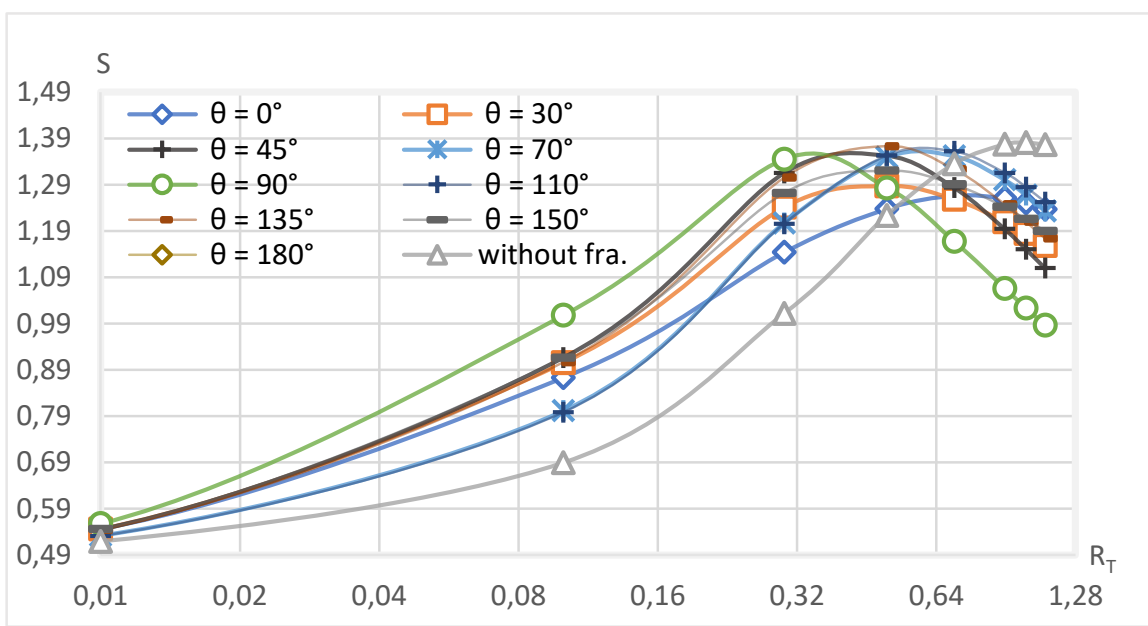

Figure 8: Effect of the fracture's orientation on the separation for $\mathrm{Le}=10, \mathrm{e}=0.038$, $S p=0.5$, and $\alpha=30$ at low Rayleigh numbers 

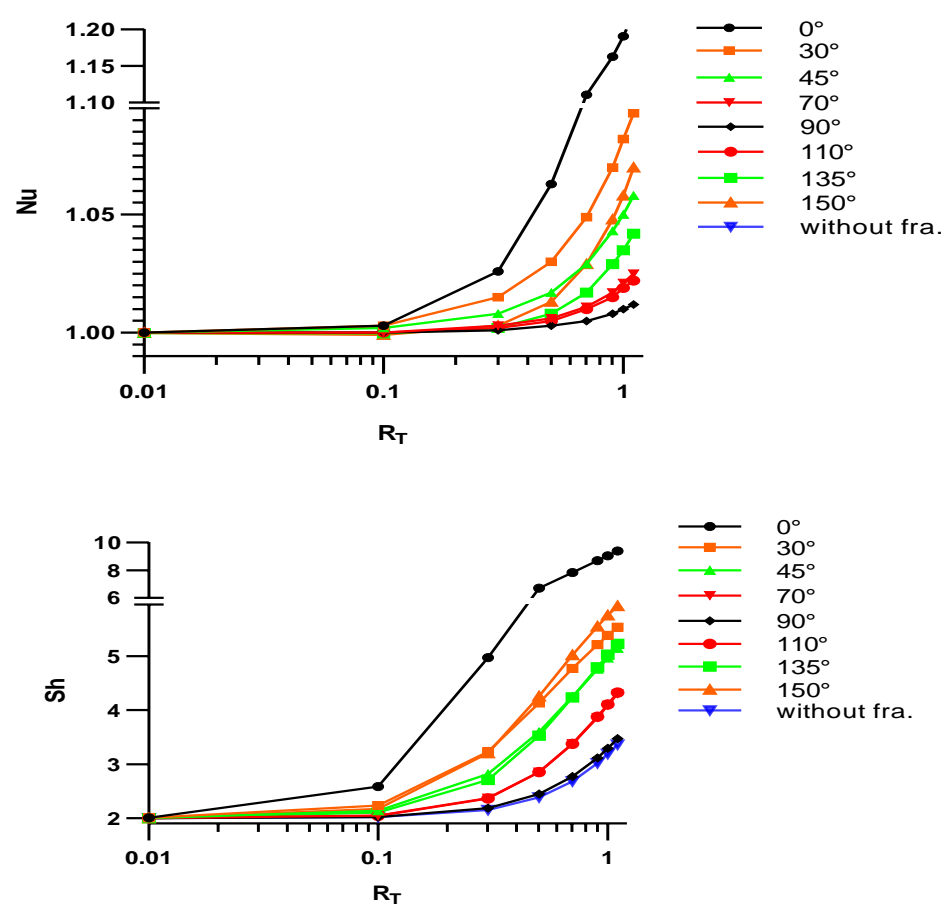

Figure 9: Nusselt $(\mathrm{Nu})$ and Sherwood $(\mathrm{Sh})$ numbers for $L e=10, e=0.038, S p=0.5$, and $\alpha=30$ at low Rayleigh numbers

The heat and mass transfer made by vertically symmetric fractures of an inclination of $\theta$ and $\pi-\theta$ is the same in most cases, but it becomes more differentiated for small $\theta$ (closely horizontal fracture). Note that the length of the fracture is variable with the angle, but it is the same for vertically symmetric fractures.

The difference between the vertically symmetric fracture behavior towards heat and mass transfer is a result of a combination of several effects including the direction of convection circulation, the gravity acceleration, boundary conditions, and the Soret parameter sign in our case, for positive Soret parameter, the mass transfer for the heavier component is made towards the cold wall (left end) by both diffusion and thermodiffusion effects. However, with the same counter clockwise convection flow direction, fractures inclined towards the cold wall (left-end) and heated from the bottom have more important convection forces than the ones inclined toward the hot wall (right-end) and heated from the top. For inclined fracture near the vertical, this difference no longer persists, for instance $\theta=70^{\circ}$, and $\theta=110^{\circ}$. 


\section{Conclusion}

Thermosolutal convection in porous media is significantly affected by the contribution of Soret effect and convection forces, where the permeability of the medium is the dominant parameter. The fracture effect on the thermosolutal convection, considering the Soret effect has been rarely studied before. In this investigation, a fractured porous medium from the center of the cavity is considered and characterized by slightly higher permeability to improve the TGC separation technique.

Deducing from the analysis carried out in this study, we conclude that the fracture in the porous medium strongly influences the behavior of the thermogravitational flow and the corresponding heat and mass transfer performance in the cavity, which has effects on the separation.

At low Rayleigh numbers $R_{T}$, the Soret effect dominates the mass transfer process, and the addition of a fracture to the porous medium plays an important role in enhancing convective forces, which leads to increase heat and mass transfer and promotes better separations (depending on its permeability and position). However, the fracture can cause a negative effect on the separation when the Rayleigh number of the porous medium is higher or equal to the optimal value.

Particular attention is given to the orientation of the fracture. It comes from the analysis, that at low Rayleigh number $R_{T}$, the more the inclination of the fracture is close to the horizontal; the more it favors the heat and mass transfer. Furthermore, due to the heating source, fractures inclined towards the cold wall have higher convection forces than the ones inclined towards the hot wall. This could increase or decrease the separation depending on $R_{T}$ and $\alpha$.

\section{References}

[1] De Groot, S. R., "L'effet Soret-diffusion thermique dans les phases condenses", Doctoral Thesis, Amsterdam, 1945.

[2] Soret, C., "Sur l'état d'équilibre que prend au point de vue de sa concentration une dissolution saline primitivement homogène dont deux parties sont portées à des températures différentes", Arch. Sci. Phys. Nat., vol. 2, pp. 48-61, 1879.

[3] Ludwig, C., "Diffusion zwischen ungleich erwarmten Orten gleich zusammengesetzer", Akad. Wien. Math. Naturwiss, vol. 20, pp. 539-539, 1856.

[4] Clusius, K. and Dickel, G., "New process for separation of gas mixtures and isotopes", Naturwissenschaften, vol. 26, pp. 546-546, 1938.

[5] Furry, W. H., Jones, R. C., and Onsager, L., "On the theory of isotope separation by thermal diffusion”, Phys. Rev., vol. 55, pp.1083-1093, 1939.

[6] DeGroot, S. R., "Theorie phénoménologique du procédé thermogravitationnel de séparation dans un liquid", Physica IX, vol. 8, pp. 801-816, 1942.

[7] Philomena, G. G. and Barbara, F., "Clusius-Dickel separation: a new look at an old technique”, Separation Science, vol. 12, no. 2, pp. 103-169, 1977. 
Thermosolutal Convection in a Porous Medium Cavity Subjected to Heat and Mass Flux 103

[8] Lorenz, M. and Emery, A. H., "The packed thermodiffusion column”, Chemical Engineering Science, vol. 11, pp. 16-23, 1959.

[9] Lee, J., Shivakumara, I. S. and Mamatha, A. L., "Effect of non-uniform temperature gradients on thermogravitational convection in a porous layer using a thermal non-equilibrium model", J. Porous Media, vol. 14, pp. 659-669, 2011.

[10] Abahri, O., Sadaoui, D., Mansouri, K., Mojtabi, A. and Mojtabi, M. C., "Thermogravitational separation in horizontal annular porous cell", Mechanics \& Industry, vol. 18, no. 1, pp. 106116, 2017.

[11] Grigor'ev, V. and Rivin, E., "Determination of the Soret coefficient based on the separation of mixtures of organic liquids in a thermogravitational column", Theoretical Foundations of Chemical Engineering, vol. 51, no. 4, pp. 464-467, 2017.

[12] Mutschler, D., Larabi, M. A. and Mojtabi, A., "Theoretical models for the thermogravitational separation process in porous media filled by n-component mixtures". The European Physical Journal E, vol. 40, no. 4, pp. 49-56, 2017.

[13] Mojtabi, A., Ouattara, B., Rees, D. A. S. and Charrier-Mojtabi, M.-C., "The effect of conducting bounding horizontal plates on species separation in porous cavity saturated by a binary mixture", Int. J. Heat Mass Transfer, vol. 126, pp. 479-488, 2018.

[14] Kozlova, S. V. and Ryzhkov, I. I., "The transient separation of multicomponent mixtures in a cylindrical thermogravitational column", Int. J. Heat Mass Transfer, vol. 126, pp. 660-669, 2018.

[15] Hashemipour, N., Karimi-sabet, J., Motahari, K., Monfared, S. M., Amini, Y., And Moosavian, M. A., "Experimental and simulation investigation on separation of binary hydrocarbon mixture by thermogravitational column", Molliq, 2018.

[16] Benano-Melly, L. B., Caltagirone, J. P., Faissat, B., Montel, F., and Costeseque, P., "Modelling Soret coefficient measurement experiments in porous media considering thermal and solutal convection", Int. J. Heat Mass Transfer, vol. 44, pp. 1285-1285, 2001.

[17] Marcoux, M. and Costeseque, P., "Study of transversal dimension influence on species separation in thermogravitational diffusion columns", Journal of Non-Equilibrium Thermodynamics, vol. 32, no. 3, pp. 289-298, 2007.

[18] Boutana, N., Bahloul, A., Vasseur, P., and Joly, F., "Soret and double diffusive convection in a porous cavity", J. Porous Media, vol. 7, no. 1, pp. 41-57, 2004.

[19] Alloui, Z., Merabtine A., and Vasseur, P., "Soret and thermosolutal effects on natural convection in a vertical cavity filled with a binary mixture", The Canadian Journal of Chemical Engineering, vol. 88, pp. 718-727, 2010.

[20] Amahmid, A., Hasnaoui, M., Mamou, M., and Vasseur, P., "On the transition between aiding and opposing double diffusive flows in a vertical porous matrix", J. Porous Media, vol. 3, pp. 123-137, 2000.

[21] Er-Raki, M., Hasnaoui, M., Amahmid, A., Mamou, M., and Bourich, M., "Soret effect on double-diffusive boundary layer flows in a vertical porous cavity", J. Porous Media, vol. 10, pp. 783-795, 2007.

[22] Jiang, C. G., Saghir, M. Z., Kawaji, M., and Ghorayeb, K., "Two-dimensional numerical simulation of thermogravitational convection in a vertical porous column filled with a binary fluid mixture", Int. Journal of Thermal Sciences, vol.43, pp. 1057-1065, 2004.

[23] Jiang, C. G., Saghir, M. Z., and Kawaji, M., "Numerical analysis of thermal-solutal convection in heterogeneous porous media", Journal of Applied Mechanics, vol. 73, pp. 21-25, 2005.

[24] Jaber, T. J., "Numerical study of permeability effect on convection in fractured porous media filled with hydrocarbon ternary mixture", Proceedings of CHT-08 ICHMT Int. Symposium on Advances in Computational Heat Transfer, Marrakesh, Morocco, May 11-16, 2008. 
[25] Charrier-Mojtabi, M. C., El Hajjar, B., Ouattara, B., Mojtabi, A., and Costesèque, P., "Soretdriven convection and separation of binary mixtures in a porous horizontal slot submitted to a heat flux", C. R. Mecanique, vol. 339, pp. 303-309, 2011.

[26] Khadiri, A., Amahmid, A., Hasnaoui, M., and Rtibi, A., "Soret effect on double-diffusive convection in a square cavity heated and salted from below", Numerical Heat Transfer, Part A, vol. 57, pp. 848-868, 2010.

[27] El Hajjar, B., "Sur le couplage Thermodiffusion - Convection: Séparation et instabilités induites par de nouvelles configurations géométriques et thermiques", Doctoral Thesis, IMFT, Toulouse, 2008.

[28] Goyeau, B., Songbe, J. P., and Gobin, D., "Numerical study of double-diffusive natural convection in a porous cavity using the Darcy-Brinkman formulation", Int. J. Heat Mass Transfer, vol. 39, pp. 1363-1378, 1996.

[29] Costesèque P., Fargue D., Jamet P., "Thermodiffusion in porous media and its consequences", in "Thermal nonequilibrium phenomena in fluid mixtures", Lecture Notes in Physics, Köhler W. and Wiegand S., Eds., Springer, Berlin, Heidelberg vol. 584, pp. 389427, 2002. 